\title{
42 Lowering quality damage in open-field vegetables caused by Meloidogyne chitwoodi and M. fallax in the Low Countries
}

\author{
Wim M.L. Wesemael ${ }^{1, \star}$ and Johnny Visser ${ }^{2}$ \\ ${ }^{1}$ Flanders Research Institute for Agriculture, Fisheries and Food (ILVO)/Ghent \\ University, Merelbeke, Belgium; ' Wageningen University \& Research, \\ Field Crops, Lelystad, The Netherlands
}

\section{Introduction}

The Low Countries (the Netherlands [NL] and Belgium [B]) provide some of the best agricultural soils in the world for open-field vegetable production rendering high yields per hectare. The processing industry for frozen and preserved vegetables has high economic value in both countries. In 2019, 18.2\% of the world's frozen vegetables came from Belgium and $4 \%$ from the Netherlands (FAOSTAT, 2021). Since the 1990s, pre-harvest quality control of carrots (Daucus carota) and black salsify (Scorzonera hispanica) showed an increase in tap root damage with severe galling and rough surface rendering the infected vegetables unprocessable. This quality damage was caused by the polyphagous root-knot nematodes Meloidogyne chitwoodi and M. fallax. Meloidogyne chitwoodi was first described on potatoes in the Pacific North-west of the USA in 1980, but re-examination of old specimens and illustrations in the Netherlands and the relatively high genetic distances between Belgian populations suggest a longer presence in the Low Countries. Meloidogyne fallax was detected for the first time in 1992 in a field near Baexem (NL) and described as a new species in 1996. Since 1998, M. chitwoodi and M. fallax have been listed as quarantine organisms in the EU (EC Directive 2000/29/EC).

\section{Distribution}

The global distribution of $M$. chitwoodi and $M$. fallax is limited (Fig. 42.1). Their presence in the Low Countries and information on their biology and life cycle are covered in Chapter 48.

\section{Symptoms of damage}

Both species can cause severe galling and rough surface rendering of infected vegetables, making them unprocessable (Fig. 42.2). Often these symptoms are caused by the second generation and they increase with longer field periods of the crop. The galls produced on lateral roots or on alternate host crops (onions, peas, spinach, leek, green beans and lettuce) that could be used in

* Corresponding author: wim.wesemael@ilvo.vlaanderen.be 


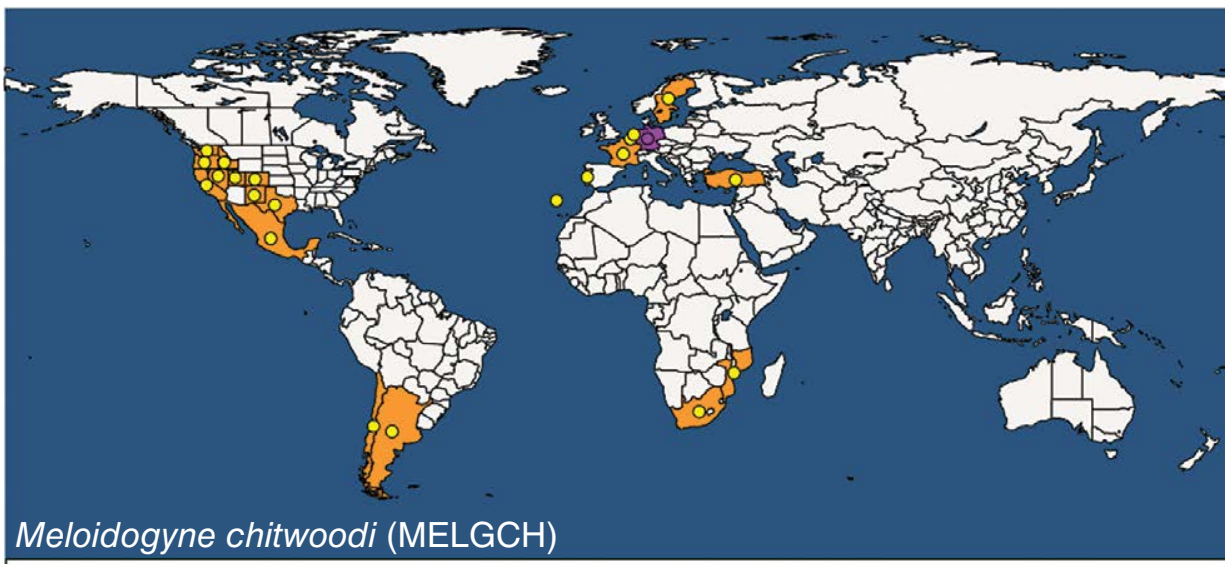

Meloidogyne chitwoodi (MELGCH)

O Present OTransient

2020-12-08

(c) EPPO https://gd.eppo.int

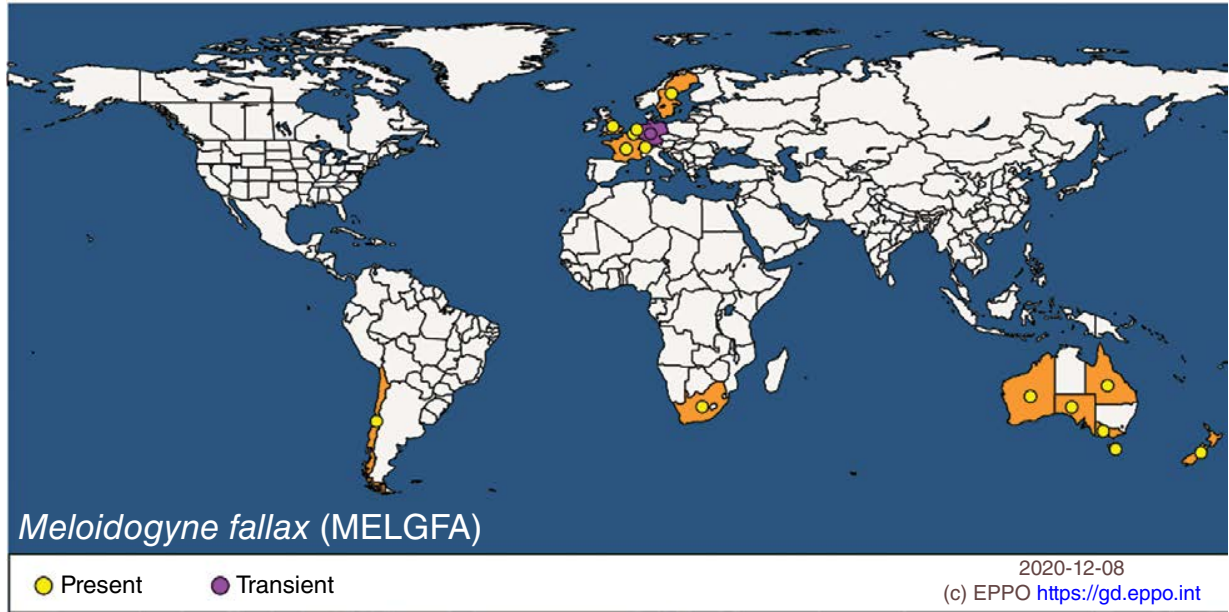

Fig. 42.1. Global distribution of Meloidogyne chitwoodi and Meloidogyne fallax. Figure courtesy of EPPO Global Database, https://gd.eppo.int (accessed 5 April 2021).
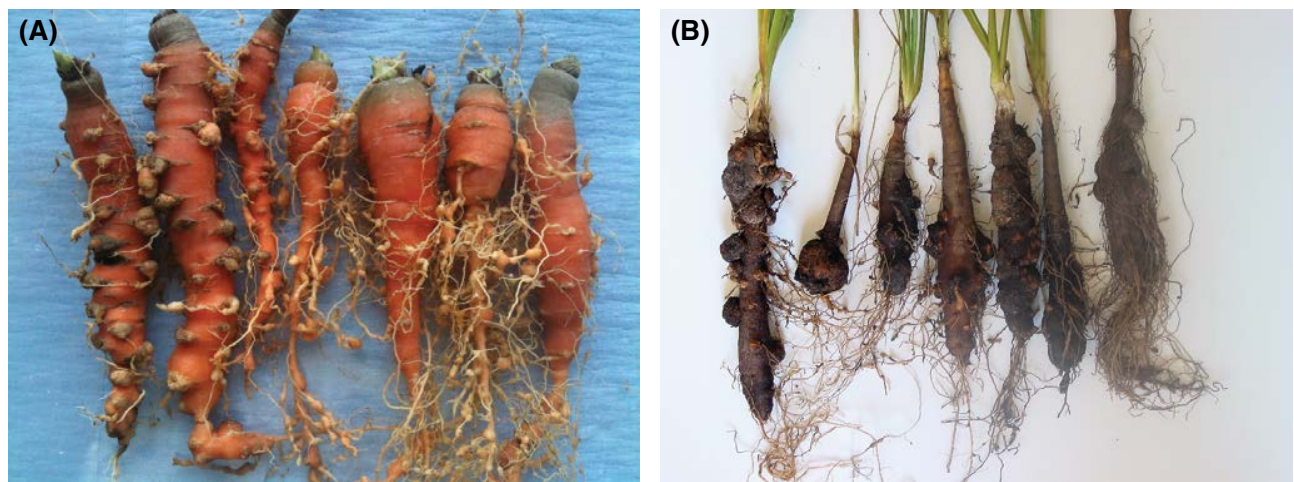

Fig. 42.2. Quality damage on (A) carrot and (B) black salsify caused by Meloidogyne chitwoodi. Photographs courtesy of W. Wesemael. 
rotations with tuber vegetables are mostly small and can be overlooked.

\section{Economic impact}

A survey in 2015 revealed that $52 \%$ of the openfield vegetable farmers in Flanders (B) were confronted with losses due to plant parasitic nematodes. Meloidogyne chitwoodi, Pratylenchus penetrans and Heterodera schachtii were reported as the major problems. At the farmers' level, economic impact due to M. chitwoodi and M. fallax is reflected in (i) direct yield loss mostly in quality loss; (ii) future loss due to spreading of the nematode to other fields with machinery; (iii) indirect losses when non-susceptible or tolerant, less profitable crops are included in the rotation; and (iv) extra costs related to phytosanitary measures due to the quarantine status.

At low initial $M$. chitwoodi densities $(\mathrm{Pi}=3$ $\mathrm{J} 2 / 100 \mathrm{~cm}^{3}$ soil), $1.5 \%$ damage on carrots was reported under field conditions (Wesemael and Moens, 2008a) leading to a financial loss of $€ 500 /$ ha. If pre-harvest quality control of carrots and black salsify show $>30 \%$ damage then the crops are no longer harvested and financial loss is $€ 2500-5500 /$ ha. When profitable vegetable crops need to be replaced by other crops due to M. chitwoodi or M. fallax, Belgian farmers often choose maize. Compared to vegetables, maize has about $€ 1400 /$ ha less financial value.

Spreading of M. chitwoodi and M. fallax puts pressure on the availability of high-quality soils for vegetable production in the vicinity of the processing industry. Allocation of the production to neighbouring countries (France, Germany, Poland) renders a 10\% higher variable cost due to increased transport times and costs. Further migration of production and processing to other countries will also have an impact on local employment.

\section{Recommended integrated nematode management}

\section{Prevention}

With increasing occurrence of unprocessable tap roots, the industry organized preventative soil sampling in collaboration with farmer associations. A contract for sensitive vegetables such as carrots and black salsify will only be given for soils that are free of M. chitwoodi and M. fallax. About 33\% of the sampled fields were rejected. Not all companies follow this strategy but it is highly recommended as it reduced the rejection of produce from $7 \%$ to less than $1.5 \%$. Major constraints of preventative soil sampling are the costs for analysis ( $>€ 100$ per sample) and the potential quarantine status of fields detected with M. chitwoodi or M. fallax. When M. chitwoodi or M. fallax are detected in a field in Belgium the infestation needs to be reported to the $\mathrm{Na}-$ tional Plant Protection Organization (NPPO). In the Netherlands, this reporting is only required when the nematodes are found in propagation material. The diagnostic lab responsible for the analysis also needs to report to its NPPO. In Belgium, the phytosanitary measures require that tubers and roots be cleaned (removal of soil) before they are allowed to leave an infested field. Also, machinery needs to be cleaned before they leave the field. These measures are cumbersome and expensive. After detection, the farmer needs to wait for 3 years before he can ask for an official re-sampling and analysis to declare the field free of $M$. chitwoodi or M. fallax. Belgian vegetable farmers mostly work with leaseholds and these can change annually. Landowners might ban farmers who report $M$. chitwoodi or M. fallax infestations and as a consequence often infestations are not reported to the NPPO. To overcome reporting by the analysing diagnostic lab, samples are sent to diagnostic services in the Netherlands or France. These labs report to their own NPPO and it takes time before the findings are shared with the Belgian NPPO. Often the exchange of information is limited to once per year and lacks details on exact location.

Harvesting is mostly done by specialized companies who move from field to field and often work 24 hours per day during the harvest period. Soil adhering to machinery is at most brushed away before leaving the field but mostly no actions are taken to avoid further spread. To minimize transportation costs, efforts are made to reduce the soil adhering to the harvested product. At the industrial processing plant this soil is collected and returned to agricultural fields. In theory the soil returns to the field where it comes from, but in reality this is difficult to control. Farmers are willing to take the soil to 
their fields as it is fertile soil and helps to overcome erosion from their fields. Waste soil is also transported to non-agricultural locations, mostly unknown to the processor. Soil present in washing water and root pieces or peelings are mostly treated before being discarded. In Flanders (B), a processing company is sanitizing the wastewater (anaerobic and aerobic) and this water is used by local farmers to irrigate their fields. Organic waste (roots, tubers, peelings) is used as animal feed or goes to a digester.

Planting material (leek, celeriac, cabbages) is not known to be a source of M. chitwoodi or M. fallax infestation. Seedlings of celeriac and cabbages are mostly grown in substrates free of nematodes but planting material for leek is often grown in seeding beds in the field. It is important that these seeding beds are checked for the presence of plant parasitic nematodes. Potatoes are often grown in rotation with vegetables and it is of paramount importance that certified seed potatoes are used to avoid introduction of M. chitwoodi and M. fallax.

\section{Crop rotation}

Due to the wide host range of M. chitwoodi and M. fallax and the high specialization of vegetable farmers, options for crop rotation are limited. At present, it is very difficult, if not impossible, to eradicate $M$. chitwoodi and M. fallax with crop rotation once it is present in the field. However, a well-planned rotation scheme can reduce the population to levels that allow susceptible cash crops to be included. Wageningen University and Research field crops (NL) developed a useful tool (www.aaltjesschema.nl/, accessed 12 November 2020) that allows farmers to check the host plant status and damage potential of a wide variety of crops and cover crops for different plant parasitic nematodes. This program is based on the vast amount of data collected over the years and available data from the literature and is updated regularly. The nematode decision tool NemaDecide predicts population development and possible damage depending on the chosen rotation and the initial population density. This allows planning for the longer term.

Chicory is a non-host for M. chitwoodi and M. fallax. Onions and peas are poor hosts and can be included in a rotation. However, pea might suffer from damage caused by the nematodes. Spinach (Spinacia oleracea) and lettuce are poor hosts for M. chitwoodi and M. fallax and due to the short field period, it can be harvested before a new generation of the nematodes is formed thereby acting as a trap crop. Asparagus is a non-host for M. chitwoodi but good host for M. fallax. Different cultivars of green beans (Phaseolus vulgaris) show reduced or delayed development of $M$. chitwoodi and were a non-host for M. fallax and can be used to lower the population (Wesemael and Moens, 2012). It is important to destroy the stubble that remains in the field immediately after harvest to avoid further development of the nematodes. In general, huge population build-ups and damage can be avoided when crops/cultivars with a short field period are chosen that are grown in early spring. Due to market demands this strategy is not always possible.

About $75 \%$ of vegetable farmers in Belgium use cover crops in their rotation, basically to improve the soil structure and to control the nitrogen balance. Most farmers are aware that cover crops can have an effect on plant parasitic nematodes but they indicate that they need better support for successful use. Only $14 \%$ of farmers use cover crops to manage nematode problems. Cover crops with resistance to $M$. chitwoodi have been developed. For example, resistant cultivars of fodder radish (Raphanus sativus) are available and have proved to be successful (Teklu et al., 2014). They are mostly sown in August/September to allow proper crop development and coverage before the temperature drops. The use of cover crops is promoted in the framework of agroecological measures. Farmers get subsidies when using mixtures of cover crops for soil health improvement. It is important that these mixtures are composed of resistant cultivars and non-hosts for M. chitwoodi.

Several weeds are host plants for M. chitwoodi and M. fallax (Kutywayo and Been, 2006), therefore it is important to have proper weed control and volunteer potato removal within the rotation to manage both nematode species.

\section{Optimization of nematode management}

Proper management starts with knowledge about the distribution of M. chitwoodi and M. fallax. The 
current quarantine status and strict phytosanitary measures are counterproductive for their management. A regulated non-quarantine status would facilitate a better approach to the problem in the Low Countries. A soil passport that combines physicochemical data, cropping history and data on pests and diseases including M. chitwoodi and M. fallax pressure can be an important step towards proper control. These data can be a guide when both farmers and the processing industry have to make decisions on crop rotations. Moreover, a clear view on problem fields may help to prevent further spread of M. chitwoodi and M. fallax. Farm machinery or vehicles can spread nematodes through adhering infested soil particles; unfortunately, cleaning of machinery is generally not practiced. If infested fields are localized, field practices on these fields can be grouped and before the machinery is used on Meloidogyne-free fields they can be cleaned thoroughly. The same strategy should be used when crops are harvested. It will only be possible to schedule this field practice on infested fields if the distance between them is short. Further development of population growth models such as NemaDecide can reduce sample costs. Implementation of a soil passport also brings responsibility to the landowner who will have to collaborate with the land user to maintain high-quality soils.

As quality damage on tubers and tap roots is caused by the second generation of $M$. chitwoodi and M. fallax, the use of crops with a short field period leads to minimum losses. Rotations with crops that have a short field period that can be grown in the spring allow the implementation of a fallow period during the warmer months of the year before a resistant cover crop is sown. During this summer fallow, M. chitwoodi and M. fallax will require a host plant for survival and their energy reserves will drop, enhancing natural decline. As an alternative for the summer fallow, Tagetes patula can be grown as a cover crop. This is a non-host for M. chitwoodi and M. fallax and actively reduces the population of Pratylenchus penetrans which also causes substantial damage to a wide variety of vegetables.

Postponing the sowing date was shown to be successful for carrots and reduced quality damage caused by M. fallax (Molendijk and Brommer, 1998). Most likely this is due to a stronger winter decline and hence a lower initial population. For M. chitwoodi this might not be successful due to differences in survival strategy
(Wesemael et al., 2006) and peaks in the population when soil temperatures increase have been observed (Pinkerton et al., 1991; Wesemael and Moens, 2008b).

The use of decision support tools and computer-controlled monitoring of degree days as well as moisture levels during crop growth will help cover-crop trap cropping by allowing the farmer to destroy the crop before a new generation is formed (see Chapter 60). Cover crops that are fast growing can be used as trap crops in between cash crops. Incorporation of the crop in the soil will also increase the organic matter content and improve the soil quality.

Recently, promising cultivars of potato and sugar beet with resistance against $M$. chitwoodi have been developed and will be available in the near future. These can be included in rotations with vegetables.

In the Netherlands, inundation has been successful to eradicate $M$. chitwoodi in infested marine clay soils. Fields in Belgium are less suitable for this technique. At present, the cost for inundation is also too high for vegetable farmers.

\section{Future research requirements}

Breeding for M. chitwoodi and/or M. fallax resistance in vegetables is limited due to the restricted market and high costs for development. Increasing knowledge on the nematode-crop interactions and related genes, combined with transcriptomic analysis and marker-assisted selection, might facilitate the development. Also, CRISPR-CAS will enable faster development of resistant crops but at present such crops are not accepted in the EU. Breeding of crops with shorter field periods can be another option as this will also reduce the need for irrigation which is more and more required in Belgium. Irrigation with sanitized wastewater will become more important. Economic feasible treatment of waste streams (soil, roots, washing water) needs to be developed to minimize further spread and allow cultivation of root and tuber crops in M. chitwoodi or M. fallax infested soil.

Further research on M. chitwoodi/M. fallaxplant combinations, population development and damage potential is needed to improve nematode decision tools that assist farmers in their rotation planning. 


\section{Outlook: anticipating future developments}

In the future, more generations of $M$. chitwoodi and M. fallax will be formed during the growing season and multiplication most likely will continue during mild winters on cover crops. Root-knot nematodes from warmer climates will find their way into our fields and increase the problem. The current mind-set to eradicate M. chitwoodi and M. fallax seems obsolete. Rather than looking at one pest or pathogen, a holistic approach in which the agroecological system is considered is needed. Looking at shifts in nematode communities, facilitated with next-generation sequencing and meta-DNA barcoding, will allow us to adjust our agricultural practices to minimize losses caused by plant parasitic nematodes. Plant resistance and tolerance in cash crops will play a key role and can be complemented with ecological control measures.

In the future, breeding programmes will produce cover crops with resistance to M. chitwoodi, allowing more options for rotations and the additional benefits of cover crops and cover-crop mixtures. In the past, cover crops were destroyed when frost appeared in autumn. Cover crops that are not $100 \%$ resistant but delay the development of $M$. chitwoodi were destroyed before new eggs were formed and acted as a trap crop. Global warming reduces the number of nights and days with freezing temperatures and as cover crops are no longer destroyed by frost this might induce an extra generation of M. chitwoodi.

Climate change also comes with new opportunities. Crops that were restricted to warmer climates can now be selected for cultivation in temperate areas. Tests with soybean, sorghum, chickpea and sweet potato in Flanders show promising results and might bring new options for M. chitwoodi and M. fallax management. Knowledge on their host plant status and susceptibility for M. chitwoodi and M. fallax is needed.

New bioagents or chemical agents are being developed to upregulate or downregulate genes that are involved in the plant-nematode interactions. These products can be applied as foliar sprays and induce systemic acquired resistance. In combination with data on infestations and precision farming, application of these products can be fine-tuned to optimize results.

\section{References}

FAOSTAT (2021) http://www.fao.org/faostat/en/\#data/QC (accessed 15 December 2020).

Kutywayo, V. and Been, T.H. (2006) Host status of six major weeds to Meloidogyne chitwoodi and Pratylenchus penetrans, including a preliminary field survey concerning other weeds. Nematology 8 , 647-657.

Molendijk, L. and Brommer, E. (1998) Postponement of sowing reduces quality damage in carrots (Daucus carota) caused by Meloidogyne fallax. Mededelingen van de faculteit Van de Landbouwwetenschappen, Universiteit Gent 63, 655-658.

Pinkerton, J.N., Santo, G.S. and Mojtahedi, H. (1991) Population dynamics of Meloidogyne chitwoodi on Russet Burbank potatoes in relation to degree-day accumulation. Journal of Nematology 23, 283-290.

Teklu, M.G., Schomaker, C.H. and Been, T.H. (2014) Relative susceptibilities of five fodder radish varieties (Raphanus sativus var. Oleiformis) to Meloidogyne chitwoodi. Nematology 16, 577-590.

Wesemael, W.M.L. and Moens, M. (2008a) Quality damage on carrots (Daucus carota L.) caused by the root-knot nematode Meloidogyne chitwoodi. Nematology 10, 261-270.

Wesemael, W.M.L. and Moens, M. (2008b) Vertical distribution of the plant-parasitic nematode, Meloidogyne chitwoodi, under field crops. European Journal of Plant Pathology 120, 249-257.

Wesemael, W.M.L. and Moens, M. (2012) Screening of common bean (Phaseolus vulgaris) for resistance against temperate root-knot nematodes (Meloidogyne spp.). Pest Management Science 68, 702-708.

Wesemael, W.M.L., Perry, R.N. and Moens, M. (2006) The influence of root diffusate and host age on hatching of the root-knot nematodes, Meloidogyne chitwoodi and M. fallax. Nematology 8, 895-902. 